\title{
Reconstruction in Different Classes of 2D Discrete Sets
}

\author{
Attila Kuba \\ Department of Applied Informatics, József Attila University, \\ Árpád tér 2, H-6720 Szeged, Hungary \\ kuba@inf.u-szeged.hu
}

\begin{abstract}
The problem of reconstruction of two-dimensional discrete sets from their two projections is considered in different classes. The reconstruction algorithms and complexity results are summarized in the case of $h v$-convex sets, $h v$-convex polyominoes, $h v$-convex 8 -connected sets, and directed $h$-convex sets. We show that the reconstruction algorithms used in the class of $h v$-convex 4 -connected sets (polyominoes) can be used, with small modifications, for reconstructing $h v$-convex 8connected sets. Finally, it is shown that the directed $h$-convex sets are uniquely reconstructible with respect to the row and column sum vectors.
\end{abstract}

\section{Introduction}

One of the most frequently studied reconstruction problems is the construction of 2 -dimensional discrete sets from their row and column sum vectors. This kind of questions can arise, for example, in electron microscopy [9, image processing [18 and radiology [16]. The general problem of reconstructing binary matrices (or, equivalently, discrete sets) has been extensively studied. Existence, consistence and uniqueness results are known (as a summary see [3]) and reconstruction algorithms are published (e.g., 6 17]). Since, in general, the number of solutions can be very high, there is an interest to reconstruct binary matrices/discrete sets having special properties. Kuba published an algorithm to reconstruct socalled two-directionally connected discrete sets [15]. Del Lungo et al. studied the reconstruction of different kinds of polyominoes [11. Recently, Chrobak and Dürr found reconstruction algorithms for special polyominoes. Since the reconstruction in certain classes can be too complex (see [19]), the classes, where the reconstruction can be performed in polynomial time, are the most important for the applications.

This paper deals with the reconstruction problem in different classes of discrete sets. The most frequently used property is some kind of discrete version of the convexity. For example, we can suppose that the elements of the discrete sets in each row and column are consecutive. Since this property itself is not enough to have a reconstruction problem which is solvable in polynomial time (see [19]), further properties can be included (e.g., connectedness). From the literature five properties are collected ( $h$-convex, $v$-convex, 4 -connected, 8-connected, and directed discrete sets) and the classes constructed from these combinations are 
studied from the viewpoint of reconstruction. As a result of this survey study, we have found classes where known algorithm, after small modifications, can be used for reconstructing discrete sets of other classes. Also, a new reconstruction algorithm is given.

First, the necessary definitions and notations are introduced. Then in the next sections the reconstruction problem in the following classes are discussed: $h v$-convex sets, $h v$-convex polyominoes, $h v$-convex 8 -connected sets, and directed $h$-convex sets.

\section{Definitions and notations}

Let $\mathbb{Z}^{2}$ denote the 2-dimensional integer lattice. In this paper the elements of $Z^{2}$ will be called points or positions. The finite subsets of $Z^{2}$ will be called discrete sets. The class of discrete sets will be denoted by $\mathcal{F}$.

Let $F$ be a discrete set. Then there is a discrete rectangle $T$ of size $m \times n(m$ and $n$ are positive integers),

$$
T=\{1, \ldots, m\} \times\{1, \ldots, n\},
$$

such that $T$ is the smallest discrete rectangle containing $F$. The discrete set $F$ can also be represented as a binary matrix $\left(f_{i j}\right)_{m \times n}, f_{i j} \in\{0,1\}$, such that

$$
f_{i j}= \begin{cases}1, & \text { if }(i, j) \in F \\ 0, & \text { otherwise }\end{cases}
$$

For any subset of $T$ we define the $i$-th row of the subset, $1 \leq i \leq m$, as its intersection with $i \times\{1, \ldots, n\}$. Similarly, the $j$-th column, $1 \leq j \leq n$, of the subset is its intersection with $\{1, \ldots, m\} \times j$.

Let $\mathbb{N}$ denote the set of positive integers. For any discrete set $F$ we define its projections by the operations $\mathcal{H}$ and $\mathcal{V}$ as follows. $\mathcal{H}: \mathcal{F} \longrightarrow \mathbb{N}^{m}, \mathcal{H}(F)=$ $H=\left(h_{1}, \ldots, h_{m}\right)$ where

$$
h_{i}=\sum_{j=1}^{n} f_{i j}, \quad i=1, \ldots, m,
$$

and $\mathcal{V}: \mathcal{F} \longrightarrow \mathbb{N}^{n}, \mathcal{V}(F)=V=\left(v_{1}, \ldots, v_{n}\right)$ where

$$
v_{j}=\sum_{i=1}^{m} f_{i j}, \quad j=1, \ldots, n .
$$

The vectors $H$ and $V$ will be called the row and column sum vectors of $F$, respectively.

Let $F$ and $F^{\prime}$ be discrete sets. We say that $F$ and $F^{\prime}$ are tomographically equivalent (w.r.t. the row and column sum vectors) if

$$
\mathcal{H}(F)=\mathcal{H}\left(F^{\prime}\right) \quad \text { and } \quad \mathcal{V}(F)=\mathcal{V}\left(F^{\prime}\right)
$$


The discrete set $F$ is unique (w.r.t. the row and column sum vectors) if there is no discrete set $F^{\prime}(\neq F)$ being tomographically equivalent to $F$.

Let $\mathcal{G}$ be a class of discrete sets. The discrete set $F \in \mathcal{G}$ is determined (by their projections) if there is no tomographically equivalent set in the class $\mathcal{G}$.

We are going to study the following problem in different classes of discrete sets.

$\operatorname{Reconstruction}(\mathcal{G})$.

Instance: $\quad$ Two vectors $H \in \mathbb{N}^{m}$ and $V \in \mathbb{N}^{n}$.

Task: $\quad$ Construct a discrete set $F \in \mathcal{G}$ such that $\mathcal{H}(F)=H$ and $\mathcal{V}(F)=V$.

Different classes of discrete sets will be introduced by the following definitions.

Let $P$ and $Q$ be two points in $Z^{2}$. Let us consider the Euclidean distance between $P$ and $Q$,

$$
\|P-Q\|=\sqrt{\left(p_{1}-q_{1}\right)^{2}+\left(p_{2}-q_{2}\right)^{2}} .
$$

The points $P$ and $Q$ are said to be 4 -adjacent if $\|P-Q\| \leq 1$ and 8-adjacent if $\|P-Q\| \leq \sqrt{2}$. (Explicitly, the 4-adjacent points of $(i, j)$ are $(i-1, j),(i, j-1)$, $(i, j+1)$ and $(i+1, j)$, i.e. the next points in directions north, west, east and south, respectively, and the point $(i, j)$ itself.) The sequence of distinct points $\left(i_{0}, j_{0}\right), \ldots,\left(i_{k}, j_{k}\right)$ is a 4 -path/8-path from point $\left(i_{0}, j_{0}\right)$ to point $\left(i_{k}, j_{k}\right)$ in a discrete set $F$ if each point of the sequence is in $F$ and $\left(i_{l}, j_{l}\right)$ is 4 -adjacent/8adjacent, respectively, to $\left(i_{l-1}, j_{l-1}\right)$ for each $l=1, \ldots, k$. Two points are 4 connected/8-connected in the discrete set $F$ if there is a 4-path/8-path, respectively, in $F$ between them. A discrete set $F$ is 4 -connected/8-connected if any two points in $F$ are 4-connected/8-connected, respectively, in $F$. The 4-connected set is also called polyomino (see, for example, Golomb [12]). The classes of 4connected and 8-connected sets will be denoted by $\left(c_{4}\right)$ and $\left(c_{8}\right)$, respectively. From the definitions it follows that $\left(c_{4}\right) \subset\left(c_{8}\right)$.

The discrete set $F$ is horizontally convex (or, shortly, $h$-convex) if its rows are 4 -connected. The class of $h$-convex sets will be denoted by $(h)$. Similarly, a discrete set $F$ is vertically convex (or, shortly, $v$-convex) if its columns are 4 -connected. The class of $v$-convex sets will be denoted by $(v)$. The $h$ - and $v$ convex sets will be called $h v$-convex and their class will be denoted by $(h, v)$. Clearly, $(h, v)=(h) \cap(v)$. Using this notation, the $h v$-convex polyominoes are the class of $\left(c_{4}, h, v\right)$.

The sequence of distinct points $\left(i_{0}, j_{0}\right), \ldots,\left(i_{k}, j_{k}\right)$ is an EN-path from point $\left(i_{0}, j_{0}\right)$ to point $\left(i_{k}, j_{k}\right)$ in a discrete set $F$ if each point of the sequence is in $F$ and $\left(i_{l}, j_{l}\right)$ is in east or north to $\left(i_{l-1}, j_{l-1}\right)$ for each $l=1, \ldots, k$. The discrete set $F$ is directed if there is a particular point of $F$, called source, such that there is an EN-path in $F$ from the source to any other point of $F$. It follows from the definition that the source point of a directed set is necessarily the point $(m, 1)$. The class of directed sets will be denoted by $(d)$. Clearly, $(d) \subset\left(c_{4}\right)$. 


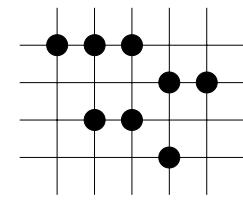

$a$

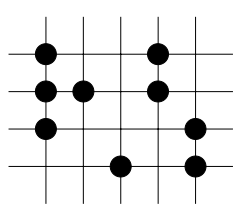

$\boldsymbol{b}$

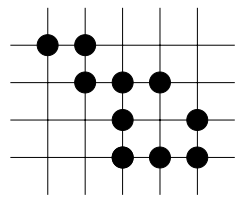

$c$
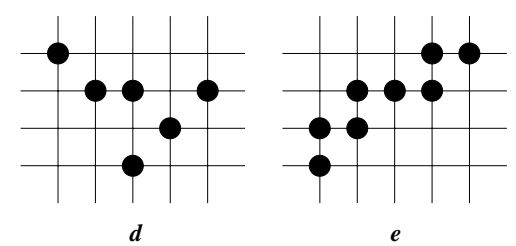

Fig. 1.Examples of discrete sets in different classes. a) $h$-convex discret set; b) $v$-convex discrete set; c) 4-connected discrete set; d) 8-connected discrete set; f) directed discrete set.

\section{Reconstruction of $h v$-convex sets}

The reconstruction of $h v$-convex sets was studied by Kuba [15]. He gave a greedy type heuristic algorithm, by which the set $F$ to be reconstructed could be approximated by two sequences of discrete sets, $\left\{K^{(l)}\right\}$ and $\left\{S^{(l)}\right\}$. The first sequence is nondecreasing and it consists of $h v$-convex sets supposed to be the subsets of $F$, called core sets. The other sequence is nonincreasing and its consists of discrete sets supposed to contain $F$, called envelope sets. As initial core and envelope sets we can use

$$
K^{(0)}=\emptyset \quad \text { and } \quad S^{(0)}=T .
$$

The core set in each iteration will be constructed from the (previous) envelope set $S$ and from the projections $H$ and $V$ as follows. First, we create the discrete set $K_{h}(S, H)$, the intersection of all $h$-convex sets contained by $S$ and having horizontal row sum $H$. (It can be computed easily row by row. For example, if the $i$-th row of $\mathrm{S}$ consists of $\left(i, s_{i}^{\prime}\right),\left(i, s_{i}^{\prime}+1\right), \ldots,\left(i, s_{i}^{\prime \prime}\right)\left(1 \leq s_{i}^{\prime} \leq s_{i}^{\prime \prime} \leq n\right)$ and $h_{i} \leq s_{i}^{\prime \prime}-s_{i}^{\prime}+1 \leq 2 * h_{i}-1$ then the $i$-th row of $K_{h}(S, H)$ is the set of $\left\{\left(i, s_{i}^{\prime \prime}-h_{i}+1\right),\left(i, s_{i}^{\prime \prime}-h_{i}+2\right), \ldots,\left(i, s_{i}^{\prime}+h_{i}-1\right)\right\}$.) Similarly, using the envelope $S$ and the column sums $V$ the set $K_{v}(S, V)$ can be defined. Clearly, if $F \subseteq S$ then $K_{h}(S, H) \subseteq F$ and $K_{v}(S, V) \subseteq F$. Therefore, we can select the intersection of all $h v$-convex sets containing $K_{h}(S, H) \cup K_{v}(S, V)$ as the new core. (See Fig. 2])

Analogously, the envelope set in each iteration will be constructed from the (previous) core set $K$ and from the projections $H$ and $V$ as follows. First, we create the discrete set $S_{h}(K, H)$, the union of all $h$-convex sets containing $K$ and having horizontal row sum $H$. (It can be computed easily row by row. For example, if the $i$-th row of $K$ consists of $\left(i, k_{i}^{\prime}\right),\left(i . k_{i}^{\prime}+1\right), \ldots,\left(i, k_{i}^{\prime \prime}\right)(1 \leq$ 


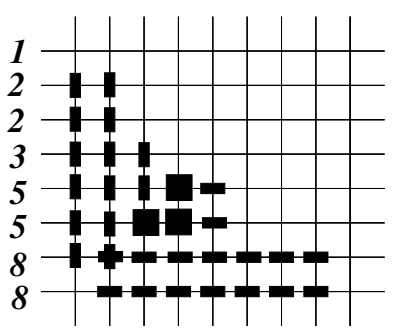

$\begin{array}{lllllllll}7 & 7 & 4 & 4 & 2 & 2 & 2 & 1\end{array}$

$a$

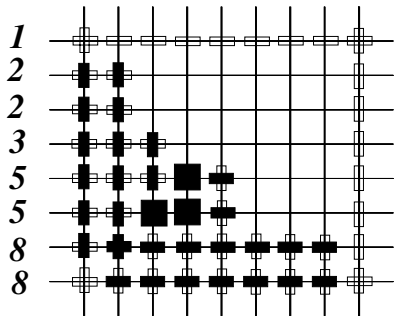

$\begin{array}{lllllllll}7 & 7 & 5 & 4 & 4 & 2 & 2 & 2 & 1\end{array}$

b

Fig. 2. Construction of core and envelope sets. a) The construction of the core set from the envelope set $S=T$ and from the projections. The elements of $K_{h}(S, H)$ and $K_{v}(S, V)$ are denoted by horizontal and vertical thick line segments, respectively. The squares denote the new elements of the intersection of all $h v$-convex sets containing $K_{h}(S, H) \cup K_{v}(S, V)$. b) The construction of the envelope set from the core set constructed in a). The elements of $S_{h}(K, H)$ and $S_{v}(K, V)$ are denoted by horizontal and vertical line segments, respectively.

$\left.k_{i}^{\prime} \leq k_{i}^{\prime \prime} \leq n\right)$ and $k_{i}^{\prime \prime}-k_{i}^{\prime}+1 \leq h_{i}$ then the $i$-th row of $S_{h}(K, H)$ is the set of $\left\{\left(i, k_{i}^{\prime \prime}-h_{i}+1\right),\left(i, k_{i}^{\prime \prime}-h_{i}+2\right), \ldots,\left(i, k_{i}^{\prime}+h_{i}+1\right)\right\}$.) Similarly, using the core $K$ and the column sums $V$ the set $S_{v}(K, V)$ can be defined. Clearly, if $K \subseteq F$ then $F \subseteq S_{h}(K, H)$ and $F \subseteq S_{v}(K, V)$. Therefore, we can select the set $S_{h}(K, H) \cap S_{v}(K, V)$ as the new envelope.

During the iterations we have

$$
K^{(l)} \subseteq F \subseteq S^{(l)}
$$

If we have $K^{(l)}=S^{(l)}$ for some $l$ then $F=K^{(l)}=S^{(l)}$ is a solution. It may also happen that during the iterations we reach a situation when core or envelope do not change or $K^{(l)} \nsubseteq S^{(l)}$. In the first case, we can take an arbitrary element of the set $S^{(l)} \backslash K^{(l)}$, add it to the core and continue the algorithm with this new core set. In the second case we have a contradiction indicating that there is no solution between the current core and envelope. Then we can return to the arbitrary selected element (if we made such a formation trial earlier), take it away from the envelope set and continue the algorithm with this reduced envelope.

\section{Reconstruction of $h v$-convex polyominoes}

This class of discrete sets was studied first by Del Lungo et al. 111. The method was improved [2|4] and the latest version is able to reconstruct $h v$-convex polyominoes in time $O\left(n m \cdot \log (n m) \cdot \min \left\{m^{2}, n^{2}\right\}\right)$.

This algorithm uses also the iterative procedure applied for $h v$-convex sets, but it is basically different in two points: selection of the initial core and handling 
the situation when the core and envelope can not be changed in the way described in Section 3 .

For the selection of the initial core the concepts of feet of polyominoes are introduced. The columns of $F$ that have element in the first row of $T$ are called the north foot of $F$, denoted by $P_{N}$. Analogous definitions can be given for the south, east, and west feet, $P_{S}, P_{E}$. and $P_{W}$, respectively. The algorithm starts with choosing the positions of the four feet. (There are at most $O\left(\min \left\{m^{2}, n^{2}\right\}\right.$ ) possible cases. Some of them can be sorted out soon at the beginning of the reconstruction.) Then the $h v$-convex polyomino containing the selected feet (as an initial core) is constructed. Using the iterative procedure described in Section 3 the core set is increased and the envelope set is decreased.

If the core and envelope sets can not be changed then the reconstruction is reformulated as a 2-Satisfiability problem (also referred to as 2SAT). Such situations can be described by a 2SAT expression, i.e., a Boolean expression in conjunctive normal form with at most two literals in each clause. The 2SAT expression is satisfiable if and only if there is an $h v$-convex polyomino solution of the reconstruction problem.

Recently, Chrobak and Dürr have found a new algorithm 8] reconstructing $h v$-convex polyominoes in $O\left(m n \cdot \min \left\{m^{2}, n^{2}\right\}\right)$ time. The basic idea of their algorithm is to rewrite the whole reconstruction problem as a 2SAT problem (see Section 5).

\section{Reconstruction of $h v$-convex 8-connected sets}

This class of discrete sets was studied first by Brunetti et al. 4]. They showed that a modification of the algorithm suggested for reconstructing $h v$-convex polyominoes can be used even in this class. The complexity of the algorithm is $O\left(n m \cdot \log (n m) \cdot \min \left\{m^{2}, n^{2}\right\}\right)$ in the worst case.

In order to show the strong connections between the classes of $h v$-convex 8 -connected sets and $h v$-convex 4-connected sets (i.e., polyominoes), we present the corresponding lemmas for both classes.

Lemma 1. Let $F$ be a hv-convex polyomino with row and column sums $H$ and V. If

$$
\sum_{l=1}^{j} v_{l} \geq \sum_{k=1}^{i-1} h_{k}, \quad \sum_{k=1}^{i} h_{k} \geq \sum_{l=1}^{j-1} v_{l}, \quad \sum_{k=1}^{i} h_{k} \geq \sum_{l=j+1}^{n} v_{l}, \quad \sum_{l=j}^{n} v_{l} \geq \sum_{k=1}^{i-1} h_{k}
$$

then $(i, j) \in F$.

Proof. See [2].

Lemma 2. Let $F$ be a hv-convex 8-connected set with row and column sums $H$ and $V$. If 


$$
\sum_{l=1}^{j} v_{l}>\sum_{k=1}^{i-1} h_{k}, \quad \sum_{k=1}^{i} h_{k}>\sum_{l=1}^{j-1} v_{l}, \quad \sum_{k=1}^{i} h_{k}>\sum_{l=j+1}^{n} v_{l}, \quad \sum_{l=j}^{n} v_{l}>\sum_{k=1}^{i-1} h_{k}
$$

then $(i, j) \in F$.

Proof. See [4].

In this section we show that the modification of the algorithm suggested by Chrobak and Dürr [8] for reconstructing $h v$-convex polyominoes can be used in the more general class of $\left(c_{8}, h, v\right)$. They showed that the reconstruction of a $h v$-convex polyomino was equivalent to the evaluation of a suitable constructed 2SAT expression. Now, we give the description of the modified algorithm following the same idea as Chrobak and Dürr. Since the statements can be proved in the same way, we omit the proofs.

We say that the discrete set $A$ is an upper-left corner region in the discrete rectangle $T$ containing $F$ if $(i+1, j) \in A$ or $(i, j+1) \in A$ implies $(i, j) \in A$. The upper-right, lower-left and lower-right regions can be defined analogously. Let $\bar{F}$ denote the complement of $F$ (in $T$ ).

Lemma 3. $F \in\left(c_{8}, h, v\right)$ if and only if

$$
\bar{F}=A \cup B \cup C \cup D,
$$

where $A, B, C$, and $D$ are disjoint corner regions (upper-left, upper-right, lowerleft and lower-right, respectively).

Proof. See [8].

Remark 1. Lemma 3 is not valid in the class $\left(c_{4}, h, v\right)$. In the class $\left(c_{4}, h, v\right)$ two further conditions should be satisfied:

(i) $(i-1, j-1) \in A$ implies $(i, j) \notin D$,

(ii) $(i-1, j+1) \in B$ implies $(i, j) \notin C$.

See Fig. 3

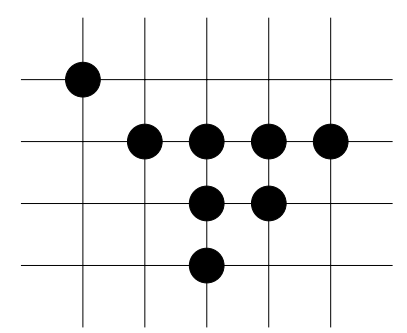

Fig. 3.An 8-connected $h v$-convex set which is not 4 -connected. 
We say that $F$ is anchored at $(k, l)(1 \leq k, l \leq m)$ if $(k, 1),(l, n) \in F$. The basic idea of the reconstruction method is to construct a 2SAT expression $F_{k, l}(H, V)$ with the property that $F_{k, l}(H, V)$ is satisfiable if and only if there is an $F \in\left(c_{8}, h, v\right)$ that is anchored at $(k, l)$. The suitable $F_{k, l}(H, V)$ in the class $\left(c_{8}, h, c\right)$ can be constructed as

$$
F_{k, l}(H, V)=\operatorname{Cor} \wedge \text { Dis } \wedge A n c \wedge L B C \wedge U B R,
$$

where $C o r$, Dis, $A n c, L B C$, and $U B R$ are sets of clauses describing the properties of "Corners", "Disjointness", "Anchors", "Lower bound on column sums", and "Upper bound on row sums", respectively, in the following way.

$$
\begin{aligned}
\text { Cor }= & \bigwedge_{i, j}\left(a_{i j} \Rightarrow a_{i-1, j} \wedge a_{i j} \Rightarrow a_{i, j-1}\right) \wedge \bigwedge_{i, j}\left(b_{i j} \Rightarrow b_{i-1, j} \wedge b_{i j} \Rightarrow b_{i, j+1}\right) \wedge \\
& \bigwedge_{i, j}\left(c_{i j} \Rightarrow c_{i+1, j} \wedge c_{i j} \Rightarrow c_{i, j-1}\right) \wedge \bigwedge_{i, j}\left(d_{i j} \Rightarrow d_{i+1, j} \wedge d_{i j} \Rightarrow d_{i, j+1}\right), \\
\operatorname{Dis}= & \bigwedge_{i, j}\left\{x_{i, j} \Rightarrow \bar{y}_{i, j} \mid \text { for symbols } X, Y \in\{A, B, C, D\}, X \neq Y\right\}, \\
\text { Anc }= & \bar{a}_{k, 1} \wedge \bar{b}_{k, 1} \wedge \bar{c}_{k, 1} \wedge \bar{d}_{k, 1} \wedge \bar{a}_{l, n} \wedge \bar{b}_{l, n} \wedge \bar{c}_{l, n} \wedge \bar{d}_{l, n}, \\
\operatorname{LBC}= & \bigwedge_{i, j}\left(a_{i j} \Rightarrow \bar{c}_{i+v_{j}, j} \wedge a_{i j} \Rightarrow \bar{d}_{i+v_{j}, j}\right) \wedge \\
& \bigwedge_{i, j}\left(b_{i j} \Rightarrow \bar{c}_{i+v_{j}, j} \wedge b_{i j} \Rightarrow \bar{d}_{i+v_{j}, j}\right) \wedge \bigwedge_{j}\left(\bar{c}_{v_{j}, j} \wedge \bar{d}_{v_{j}, j}\right), \\
U B R= & \bigwedge_{j}\left(\bigwedge_{i \leq \min \{k, l\}}\left(\bar{a}_{i, j} \Rightarrow b_{i, j+h_{i}}\right) \wedge \bigwedge_{l \leq i \leq k}\left(\bar{a}_{i, j} \Rightarrow d_{i, j+h_{i}}\right)\right) \wedge \\
& \bigwedge_{j}\left(\bigwedge_{k \leq i \leq l}\left(\bar{c}_{i, j} \Rightarrow b_{i, j+h_{i}}\right) \wedge \bigwedge_{\max \{k, l\} \leq i}\left(\bar{c}_{i, j} \Rightarrow d_{i, j+h_{i}}\right)\right) .
\end{aligned}
$$

Remark 2. In the case of $h v$-convex polyominoes one more set of clauses was included describing the "Connectivity" (more exactly, the 4-connectivity) of $F$ as

$$
\text { Con }=\bigwedge_{i, j}\left(a_{i j} \Rightarrow \bar{d}_{i+1, j+1} \wedge b_{i j} \Rightarrow \bar{c}_{i+1, j-1}\right) .
$$

Then the reconstruction algorithm can be given as

Algorithm 1 for reconstructing $h v$-convex 8-connected sets

Input. Two vectors, $H$ and $V$. (It is supposed that $H \in \mathbb{N}^{m}$ and $V \in \mathbb{N}^{n}$, $\sum_{i} h_{i}=\sum_{j} v_{j}, 1 \leq h_{i} \leq n$ for all $i=1, \ldots, m, 1 \leq v_{j} \leq m$ for all $j=1, \ldots, n$, and $m \leq n$.) 
Step 1. for $k, l=1, \ldots, m$

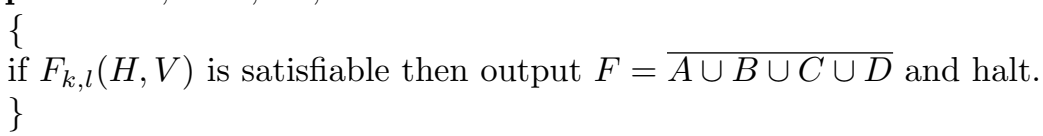

Output. print "No solution".

Theorem 1. $F_{k, l}(H, V)$ is satisfiable if and only if there is an $F \in\left(c_{8}, h, v\right)$ having projections $H$ and $V$ and it is anchored at $(k, l)$.

Proof. See [8].

Theorem 2. Algorithm 1 solves the reconstruction problem for 8-connected hvconvex sets in time $O\left(m n \cdot \min \left\{m^{2}, n^{2}\right\}\right)$.

Proof. See [8].

\section{Reconstruction of directed $h$-convex sets}

The class of directed $h v$-convex polyominoes was studied by Del Lungo et al. in [11].

In this section we consider a more general class, the class of directed $h$-convex sets, $(d, h)$. The following reconstruction algorithm is a straight consequence of the definition of the class.

Algorithm 2 for reconstructing directed $h$-convex sets

Input. Two vectors, $H$ and $V$. (It is supposed that $H \in \mathbb{N}^{m}$ and $V \in \mathbb{N}^{n}$, $\sum_{i} h_{i}=\sum_{j} v_{j}, 1 \leq h_{i} \leq n$ for all $i=1, \ldots, m, 1 \leq v_{j} \leq m$ for all $j=1, \ldots, n$, and $m \leq n$.)

Step 1. Let $B=(0)_{m \times n}$ be the initial binary matrix representing the discrete set to be reconstructed.

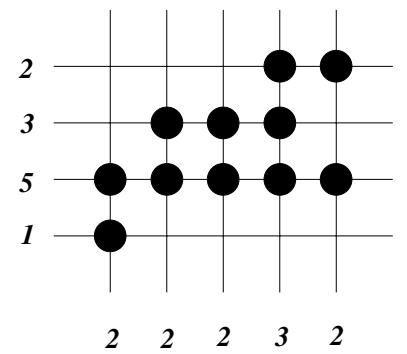

Fig. 4.Reconstruction of a directed $h$-convex set 
Step 2. for $j=1, \ldots, n$

\{ if $v_{j}<\sum_{i=1}^{m} b_{i j}$ then halt (no solution);

otherwise let $i^{\prime}$ is the maximal row index where $\sum_{l=1}^{j-1} b_{i^{\prime} l}<h_{i^{\prime}}$ (if there is no such row then halt (no solution)) and start $v_{j}-\sum_{i=1}^{m} b_{i j}$ number of rows (in rows $i^{\prime}, i^{\prime}+1, \ldots$ ) of 1 's from the column $j$ consecutively. \}

Theorem 3. If $F$ is a directed $h$-convex set then it is reconstructed by Algorithm 2.

Proof. It follows from the definition of directed $h$-convex sets.

It is also clear that there is at most one directed $h$-convex set with given projections. Finally we can remark that similar algorithm and theorem can be given for directed $v$-convex sets.

\section{Acknowledgments}

This work was supported by FKFP 0908/1997 Grant of the Hungarian Ministry of Education.

\section{References}

1. Barcucci, E., Del Lungo, A., Nivat, M., Pinzani, R.: Reconstructing convex polyominoes from horizontal and vertical projections. Theor. Comput. Sci. 155 (1996) 321-347

2. Barcucci, E., Del Lungo, A., Nivat, M., Pinzani, R.: Medians of polyominoes: A property for the reconstruction. Int. J. Imaging Systems and Techn. 9 (1998) -

3. Brualdi, R. A.: Matrices of zeros and ones with fixed row and column sum vectors. Lin. Algebra and Its Appl. 33 (1980) 159-231

4. Brunetti, S., Del Lungo, A., Del Ristoro, F., Kuba, A., Nivat, M.: Reconstruction of 8- and 4-connected convex discrete sets from row and column projections. (1998) submitted for publication

5. Chang, S.-K.: The reconstruction of binary patterns from their projections. Commun. ACM 14 (1971) 21-25

6. Chang, S.-K.: Algorithm 445. Binary pattern reconstruction from projections. Commun. ACM 16 (1973) 185-186

7. Chang, S.-K., Shelton, G. L.: Two algorithms for multiple-view binary pattern reconstruction. IEEE Trans. Systems, Man and Cybern. SMC-1 (1971) 90-94

8. Chrobak, M., Dürr, C: Reconstructing hv-convex polyominoes from orthogonal projections. (1998) submitted for publication

9. Crewe, A. V., Crewe, D. A.: Inexact reconstruction: Some improvements. Ultramicroscopy 16 (1985) 33-40

10. Del Lungo, A.: Polyominoes defined by two vectors. Theor. Comput. Sci. 127 (1994) 187-198 
11. Del Lungo, A., Nivat, M., Pinzani, R.: The number of convex polyominoes reconstructible from their orthogonal projections. Discrete Math. 157 (1996) 65-78

12. Golomb, S. W.: Polyominoes. Schribner, New York (1965)

13. Herman, G. T., Kuba, A. (Eds.): Discrete Tomography, Special Issue. Int. J. Imaging Systems and Techn. 9 (1998) No. 2/3

14. Huang, L.: The reconstruction of uniquely determined plane sets from two projections in discrete case. Techn. Report, University of Tokyo UTMS 95-29 (1995)

15. Kuba, A.: The reconstruction of two-directionally connected binary patterns from their two orthogonal projections. Comp. Vision, Graphics, and Image Proc. 27 (1984) 249-265

16. Prause, G. M. P., Onnasch, D. G. W.: Binary reconstruction of the heart chambers from biplane angiographic image sequences. IEEE Trans. Medical Imaging MI-15 (1996) 532-546

17. Ryser, H. J.: Combinatorial properties of matrices of zeros and ones. Canad. J. Math. 9 (1957) 371-377

18. Schilferstein, A. R., Chien, Y. T.: Switching components and the ambiguity problem in the reconstruction of pictures from their projections. Pattern Recognition 10 (1978) 327-340

19. Woeginger, G. W.: The reconstruction of polyominoes from their orthogonal projections. Techn. Report, Technische Universität Graz 65 (1965) 